\title{
Incorporación de la participación ciudadana en el aseguramiento de la calidad de un nomenclátor de topónimos
}

\author{
Incorporating citizens' participation to ensure the quality of a placename gazetter
}

\section{David Portolés Rodríguez (1), Rafael Martínez Cebolla (1), Fernando Germán López Martín (2), Sergio Monteagudo LatorRe (2)}

(1) Idearium Consultores, S. L., España, rafael.martinez@gmail.com; dportoles@idearium-consultores.com; (2) Departamento de Política Territorial e Interior, Centro de Información Territorial de Aragón, Gobierno de Aragón, España, \{flopezm, smonteagudo, eparaiso \}@aragon.es

\begin{abstract}
Resumen
Lograr una recopilación de nombres de lugar estandarizados, normalizados y oficiales ha sido una constante preocupación entre los gestores de información geográfica. Los topónimos constituyen una pieza clave en una Infraestructura de Datos Espaciales (IDE), ya que proporcionan la identificación inequívoca de los lugares en el territorio. Su difusión y aplicación mediante nomenclátores se ha extendido a todos los ámbitos del conocimiento y constituyen un inestimable patrimonio cultural para la entidad territorial a la que se refieren. El buscador web de nombres geográficos del Sistema de Información Territorial de Aragón (SITAR) presenta una doble visión: la difusión de los topónimos recopilados por el Gobierno de Aragón, así como una herramienta de participación ciudadana para la revisión y mejora continua de la calidad de los mismos.
\end{abstract}

Palabras clave: Nombres geográficos. Toponimia. Infraestructura de datos espaciales. Nomenclátor. Información geográfica. Corresponsabilidad administración pública-ciudadano. Gobierno abierto. Participación ciudadana.

\section{Introducción}

Históricamente, una de las principales preocupaciones de los gestores de información geográfica ha sido la de conseguir recopilar un conjunto nombres de lugar que cumplan unos determinados estándares de normalización para que puedan ser considerados como oficiales. Su complejidad en la recolección debido a la vasta colección de posibles fuentes, su adecuada gestión tecnológica para representar las relaciones posibles entre sí, así como todas las posibles variaciones que concurren en los nombres de lugar, suponen un reto para investigadores de diferentes campos de trabajo, ya sea la lingüística, la filología, la sociología, la estructura económica o la informática (Fernández, 1981).

\begin{abstract}
Achieving a collection of standardized, normalized and official place names have been a constant objective for geographic information managers. Place names are a key component of a Spatial Data Infrastructure (SDI), because they provide a unique identification for each geographic feature. Their diffusion and application via gazetteers have been extended over all knowledge areas and have become an invaluable cultural heritage of the referred territorial entity. This paper introduces a place name web search application of Territorial Information System of Aragon (SITAR), which provides a double perspective: on the one hand, it provides the dissemination of place names hosted and compiled by the Government of Aragón (a Spanish autonomous region); and on the other hand, it provides a web tool for citizen participation in order to review and improve quality of compiled place names.
\end{abstract}

Keywords: Geographical names. Place names. Spatial data infrastructure. Gazetteer. Geographic information. Shared responsibility between the Public Sector and citizens. Open Government. Citizen participation.

De entre los elementos que conforman una Infraestructura de Datos Espaciales (IDE), los nombres de lugar o topónimos destacan sobre el resto debido a que proporcionan la identificación inequívoca de los lugares en el territorio. La difusión y aplicación de los topónimos mediante nomenclátores no ha quedado restringida al ámbito geográfico, sino que se ha extendido a todos los ámbitos del conocimiento. Además, esta colección de nombres de lugar constituye un inestimable patrimonio cultural para la entidad territorial a la que hacen referencia. Sólo logrando una descripción detallada de la ubicación geográfica de cada topónimo se consigue una base sólida sobre la que es posible construir otras aplicaciones derivadas (buscadores, catálogos, visores, etc.). Por este motivo, nume- 
rosas iniciativas de organización y coordinación internacional en la gestión de los topónimos se han puesto en marcha con la aspiración común de lograr una recopilación lo más extensa posible, normalizada y oficial. Así por ejemplo, cabe destacar en este sentido a la Comisión Especializada de Nombres Geográficos (CENG), integrada en el Consejo Superior Geográfico, al Grupo de Expertos de Naciones Unidas en Nombres Geográficos (UNGEGN) o el grupo de Terminología del Consejo Internacional de Ciencias Onomásticas (ICOS).

Sin embargo, pese a la acertada intención de lograr un único conjunto de datos oficiales y normalizados, no se puede dejar de lado a todos aquellos topónimos que, no siendo considerados oficiales, tienen cierto grado de validez para un subconjunto de ciudadanos. El paso del tiempo y la etimología popular han podido deformar el léxico de algunos topónimos, pero no por ello hay que descartar estos topónimos nooficiales. Es necesario reseñar e incidir en que el topónimo en sí es un elemento a preservar sobre todo teniendo en cuenta el éxodo rural (Ayuda Bosque, 2000) de la población (en este caso el de la aragonesa) que ha abandonado el campo y se ha llevado consigo una parte del patrimonio etnográfico que es el nombre que se da a los lugares.

Para lograr esta tarea de recoger todos los topónimos, incluidos los que tienen un grado de difusión reducido, es indispensable en última instancia la participación de la sociedad para lograr un consenso mayor; y sólo se podrá conseguir esta participación si se ponen a su disposición cauces adecuados, simples y cómodos de utilizar. Articular e involucrar a todos los interesados en esta tarea es uno de los principales objetivos de todo gobierno que aspire a ser transparente y abierto.

A continuación se va a presentar el buscador web de nombres geográficos del Sistema de Información Territorial de Aragón (SITAR) como resultado de los trabajos realizados por el Gobierno de Aragón en los últimos años. Este buscador presenta una doble visión: la difusión de los topónimos recopilados por el Gobierno de Aragón, así como una herramienta de participación ciudadana para la revisión y mejora continua de la calidad de los mismos.

\section{Proceso de recopilación de topónimos}

\author{
2.1. Estado de la cuestión: \\ Los topónimos en cifras
}

En el Nomenclátor Geográfico de Aragón se dispone actualmente de un total de 621.157 nombres citados en distintas fuentes, de los cuales 484.726 están georreferenciados. Estos nombres hacen referencia a 354.872 entidades geográficas diferentes, de los cuales 286.999 tienen coordenadas, 67.873 no tienen $y$, de éstos últimos, hay 3.055 de los que no se sabe ni tan siquiera un municipio de referencia. Los nombres han sido recopilados a partir de 104 fuentes diferentes. Se dispone además de 109 fuentes adicionales pendientes de ser procesadas para incorporar nuevos topónimos. La lista de las fuentes consultadas puede obtenerse a través de: http://idearagon.es/toponimia/fuentes. htm.

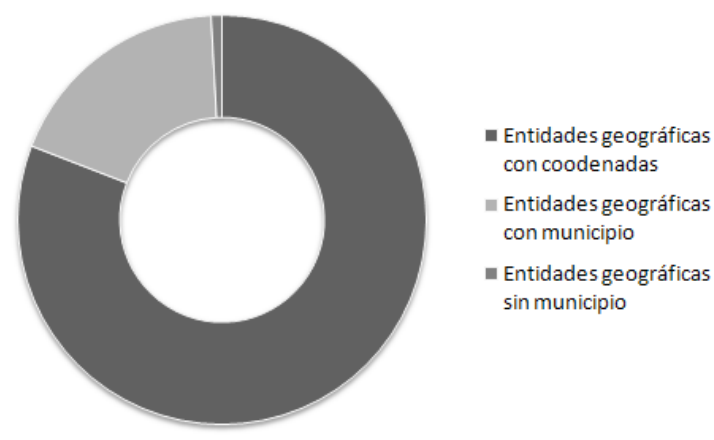

Figura 1. Distribución de entidades geográficas con coordenadas, sólo municipio y sin municipio

\subsection{Fases en la recopilación de topónimos a} partir de las fuentes

El proceso de recopilación de topónimos a partir de las distintas fuentes se ha realizado en varias fases. En primer lugar, se ha tomado como base aquellas fuentes disponibles en formato digital, priorizando entre ellas a las más completas. A este respecto, destacan principalmente los topónimos del Mapa Topográfico Nacional 1:25.000, del Nomenclátor Geográfico Básico de España NGBE_Provisional (del Instituto Geográfico Nacional, extraído de la Base Cartográfica Numérica 1:25.000) y de las entidades administrativas detalladas en el Nomenclátor 2008 de Aragón del Instituto Aragonés de Estadística.

Con esta primera selección de fuentes, se obtuvo el conjunto de topónimos más relevantes, convirtiéndose en el primer corpus toponímico con el que se comenzó a trabajar (más de 100.000 topónimos). Desde este punto de parti- 
da, se incorporó a continuación la información obtenida a partir de datos catastrales, ya sea mediante la rotulación en planos catastrales o mediante la descripción del paraje en los datos alfanuméricos asociados a una parcela, con lo que se logró otro conjunto de 100.000 topónimos adicionales. Al finalizar esta fase, se integró esta base de datos preliminar de topónimos como buscador dentro de las aplicaciones cartográficas del SITAR (Visor 2D y Cartoteca).

El punto de inflexión fue la inclusión como topónimos de las descripciones de lugar proporcionadas por los agricultores (conocedores de primera mano del territorio) en las declaraciones anuales que realizan de la PAC (Política Agrícola Común), junto con sus coordenadas geográficas. Este fue un aporte muy valioso, ya que proporcionó por un lado un gran número de topónimos no recogidos hasta la fecha en ninguna publicación oficial, y por otro lado también supuso una fuente valiosa de variedades lexicográficas que, si bien pueden no corresponder a denominaciones oficiales - diversas variaciones o incluso errores ortográficos- lo cierto es que son nombres del habla viva en la zona, que se utilizan por parte de algunos ciudadanos que viven en ese lugar, a los cuales no se desea excluir.

Sobre otras fuentes bibliográficas, se realizó un proceso de escaneado e interpretación automática de texto mediante técnicas OCR para terminar de completar la base de datos. En esta fase se han incluido principalmente estudios específicos de ámbitos territoriales concretos y denominaciones de lugares en desuso, como por ejemplo, antiguos nombres de calles cuya denominación ha cambiado a lo largo del tiempo o toponimia medieval e islámica (Ubieto Arteta, 1972, 1984; Benito Moliner et al., 2000). Este ha sido uno de los procesos más laboriosos y costosos en esfuerzo y tiempo, muy variable en función del grado de acierto de la técnica OCR y del estado de conservación y encuadernación del original. Sin embargo, ha permitido completar un gran conjunto de topónimos referidos a zonas de reducida extensión territorial. Es por este motivo por lo que se explica que la densidad de distribución de los topónimos en el territorio sea muy variable, habiendo en algunos casos un gran número en un municipio o comarca y muchos menos en otro.

Por último, cabe destacar que se han recogido también topónimos a partir de colecciones de datos disponibles en diferentes unidades administrativas del Gobierno de Aragón, tales como el catálogo de montes, el inventario de humedales singulares, vías pecuarias, bienes de interés cultural, callejero, entre otros.

\subsection{Organización territorial de los topónimos}

Los topónimos recopilados se categorizan y agrupan conforme al municipio actual al que pertenecen, si bien es cierto que no siempre es posible asignarles esta referencia geográfica indirecta. Para los topónimos sin una ubicación precisa - coordenadas-, se realiza una investigación profunda para lograr al menos asignarles el municipio vigente al que pertenecen. De este modo, no sólo se consigue que la gran mayoría tengan una referencia geográfica, aunque no sea directa o precisa; sino que además también queda organizado uno de los procesos de validación posterior que se describe en el siguiente apartado. Si se desea acceder y consultar al listado de los topónimos agrupados por municipio puede consultarse la siguiente dirección web: http://idearagon.es/toponimia/lismun. $\mathrm{htm}$.

No obstante, en algunos casos resulta imposible ubicar algunos topónimos ni siquiera en un municipio. Tal es el caso de topónimos con un ámbito de referencia territorial claramente supramunicipal - una comarca, un accidente geográfico (río, cordillera, etc.) — o aquellos topónimos que provienen de fuentes históricas con varios siglos de antigüedad y que hacen referencia a elementos geográficos que ya no existen hoy en día y para los que no se ha podido determinar ni tan siquiera una ubicación aproximada.

\subsection{Clasificaciones de topónimos recopilados}

Los topónimos, además de ser asignados a un municipio actual, son clasificados conforme a varios criterios. En primer lugar, se dividen en dos grandes bloques: toponimia más relevante (similar al tradicional concepto de toponimia mayor) y resto de toponimia (equiparable al de toponimia menor). Esta primera clasificación permite que con un subconjunto reducido de nombres se cubra un amplio porcentaje de los más utilizados, quedando el segundo grupo para una utilización más precisa y que requiera un mayor nivel de detalle. En primera instancia, se han categorizado como topónimos del primer grupo a aquellos que aparecen en una fuente muy completa y distribuida de forma homogénea en el territorio; concretamente se han seleccionado los incluidos en el mapa topográfico 1:25.000.

A posteriori se espera realizar un análisis informático, a través de estadísticas web, de los topónimos más buscados para modificar esta categorización inicial e incluir en el primer grupo a los topónimos más populares. A este respecto además se van a tomar en consideración las técnicas utilizadas por los investigadores en 
buscadores web para analizar el comportamiento del usuario ante los posibles resultados que se le ofrecen, para priorizar los que sean más seleccionados (Jones, 2006).

Otra clasificación que se ha llevado a cabo es organizarlos desde el punto de vista temático, en virtud de la cual se organizan los topónimos en hidrónimos, limnónimos, odónimos, orónimos, litónimos, antropónimos (epónimos, hagiónimios, etnónimos, endónimos o exónimos), si bien es cierto que esta clasificación sólo se está utilizando para ampliar la información al usuario y no como criterio de búsqueda.

\section{Fases del proceso de validación}

A lo largo del proceso de recopilación de topónimos se han realizado o se han previsto realizar varias fases de validación y control, de aseguramiento de la calidad de la información. A continuación se describen estas fases, las cuales se solapan habitualmente en el tiempo y se repiten iterativamente para lograr un proceso de mejora continua y perfilar el Nomenclátor Geográfico de Aragón.

\subsection{Validación automática}

El primer filtro que se realiza corresponde con validaciones automáticas que intentan detectar posibles topónimos duplicados, sinonimias, homonimias, exonimias, topónimos fuera del ámbito de la Comunidad autónoma o simples errores de carácter tipográfico. Este es el proceso más eficaz en términos de rendimiento debido a que con una pequeña intervención humana se logra una alta tasa de aciertos, descargando la mayor parte del trabajo en procesamiento computacional de máquina.

En esta fase se procede también a la normalización de la denominación de los topónimos, teniendo presentes las directivas marcadas desde otras organizaciones, tales como el Modelo de Nomenclátor de España v1.0 del Instituto Geográfico Nacional o el Manual para la normalización nacional de los nombres geográficos del Grupo de Expertos de las Naciones Unidas en Nombres Geográficos (Kerfoot, 2007).

\subsection{Validación interna}

El conjunto de topónimos se pone a disposición de otros organismos diferentes al Centro de Información Territorial de Aragón (CINTA) pero pertenecientes a la Administración Pública- para que realicen validaciones de los topónimos. Dentro del Gobierno de Aragón, el órgano encargado de aprobar el Nomenclátor Geográfico de Aragón será el Gobierno de Aragón a propuesta del Consejo de Cartografía de
Aragón. En este Consejo están representados todos y cada uno de los organismos públicos y unidades administrativas del Gobierno de Aragón con relación directa con la información geográfica. Además, se enviará la documentación para su revisión por parte del Consejo Superior de las Lenguas de Aragón y del Consejo Superior Geográfico (CSG).

\subsection{Validación externa}

Una vez realizada la validación interna y automática, se ha procedido a una nueva fase en la que se ha involucrado a los siguientes actores. Por un lado, a aquellos agentes relacionados directamente desde el punto de vista científico (autores de las fuentes toponímicas $u$ organismos especializados) y, por otro lado, a los municipios que conforman la Comunidad Autónoma de Aragón. Para ello, se les ha notificado del alcance del proyecto y de la existencia de una herramienta informática denominada "buscador de topónimos" así como un manual de utilización mediante la cual cada uno de los Secretarios de los Ayuntamientos pueden emitir las observaciones y correcciones que estimen oportunas, valiéndose de su conocimiento detallado del terreno de su competencia. En el siguiente apartado será descrita con mayor detalle.

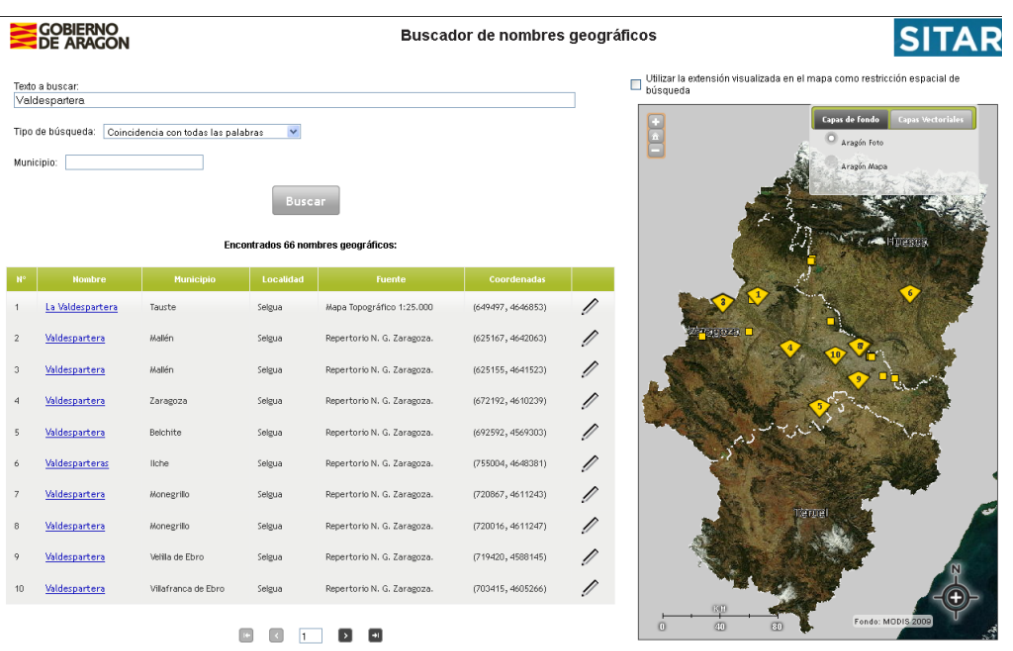

Figura 2. Imagen del buscador de nombres geográficos con notificación de cambios

Si bien es cierto que, en lo posible, se ha intentado articular que los Ayuntamientos utilicen la herramienta como cauce de validación, para aquellos no tan familiarizados con las nuevas tecnologías, se genera además un listado y un mapa correspondiente a cada uno de los respectivos municipios, para que no exista una 
barrera tecnológica que impida su participación en el proceso de mejora deseado.

En los próximos meses se espera recibir la respuesta de los ayuntamientos y se procederá a trasladar de forma manual las respuestas remitidas en formato papel al conjunto de topónimos almacenados en el nomenclátor. Las incidencias notificadas telemáticamente serán validadas directamente conforme se vayan recibiendo en la plataforma.

\subsection{Validación social: el ciudadano}

Como ya se ha mencionado, la herramienta "buscador de topónimos" es el mecanismo de validación principal y se espera que aporte el mayor número de solicitudes de mejora. Concebida como una aplicación web tradicional de búsqueda de nombres geográficos, la herramienta permite además a usuarios registrados en la plataforma la posibilidad de corregir cualesquiera de los campos almacenados en la base de datos de cada uno de los topónimos, incluida la posición de la coordenada de referencia. Para aquellos topónimos que no tengan coordenada, se puede proponer una posible ubicación, ya sea tecleando directamente las coordenadas si se conocen o bien haciendo click sobre el mapa de Aragón.

\begin{tabular}{|c|c|c|}
\hline \multicolumn{2}{|c|}{ Actualización de datos } & $x$ \\
\hline \multirow{2}{*}{$\begin{array}{l}\text { ID Entidad: } \\
\text { Nombre: }\end{array}$} & 13678 & \\
\hline & La Valdespartera & \\
\hline \multirow{2}{*}{$\begin{array}{l}\text { Municipio: } \\
\text { Localidad: }\end{array}$} & Tauste & \\
\hline & Selgua & \\
\hline \multirow{3}{*}{$\begin{array}{c}\text { Fuente: } \\
\text { Coordenada } X: \\
\text { Coordenada Y: }\end{array}$} & Mapa Topográfico 1:25.000 & \\
\hline & 649497.077 & \\
\hline & 4646853.037 & \\
\hline \multicolumn{3}{|l|}{ Comentarios: } \\
\hline & Aceptar & Cancelar \\
\hline
\end{tabular}

Figura 3. Detalle de la ventana de notificación de cambios en los campos de los topónimos

Se ha diseñado esta herramienta como la vía principal de recepción de cambios debido a que está plenamente integrada con la base de datos del SITAR que almacena la información geográfica digital y, por tanto, es más rápido y simple el hecho de validar y aceptar los cambios frente a los notificados en formato papel.

\section{Entorno tecnológico y funcionalidad del buscador}

La herramienta que se acaba de presentar hace uso de tecnologías estándar habituales en una IDE. Concretamente, la aplicación realiza consultas a un servicio Web Feature Server Gazeetteer (WFS-G) estándar que cumple con las especificaciones definidas por el Open Geospatial Consortium (OGC). El modelo de datos subyacente está basado en el Modelo de Nomenclátor de España (MNE) y en ISO 19112, con una extensión que permite la búsqueda por topónimos con variantes lexicográficas.

Estas variantes lexicográficas permiten que un usuario localice posibles resultados a partir de un conjunto de palabras clave que no están en el topónimo candidato de forma literal y exacta, pero que tienen cierta relación de semejanza (son homófonas, por ejemplo). De este modo se consigue que las variantes lexicográficas posibles que pueda aportar un usuario no supongan una barrera que impida que logre localizar los resultados que esperaba obtener (de este modo, por ejemplo, en los buscadores se encontrarán los mismos resultados tanto si se busca valdelinares como baldelinares).

La herramienta hace uso de otros componentes ya existentes en el SITAR como son los servicios web de mapas conformes al estándar Web Map Server (WMS) del OGC. De este modo, se puede alternar el fondo entre un mapa topográfico (AragonMapa) o una imagen de satélite $u$ ortofoto (AragonFoto), mientras se combina también sobre ellos la información vectorial ofrecida por el servicio WMS AragonWMS.

La interfaz gráfica presenta como elemento más destacado una única caja de texto, si bien es cierto que se proporciona además un mapa para el caso de que el usuario conozca y desee restringir la búsqueda a una determinada extensión territorial. El objetivo principal del diseño de la aplicación es el de no obligar a facilitar información previa, que el usuario no siempre conoce (Hernández, 2008), sino que sea lo más simple posible.

El desarrollo informático está basado en componentes de código abierto - a partir de los cuales se han generado desarrollos propiosdestacando principalmente Deegree y PostGIS para la implementación de la parte servidor WFS-G, así como Openlayers y JQuery para el desarrollo del cliente. 


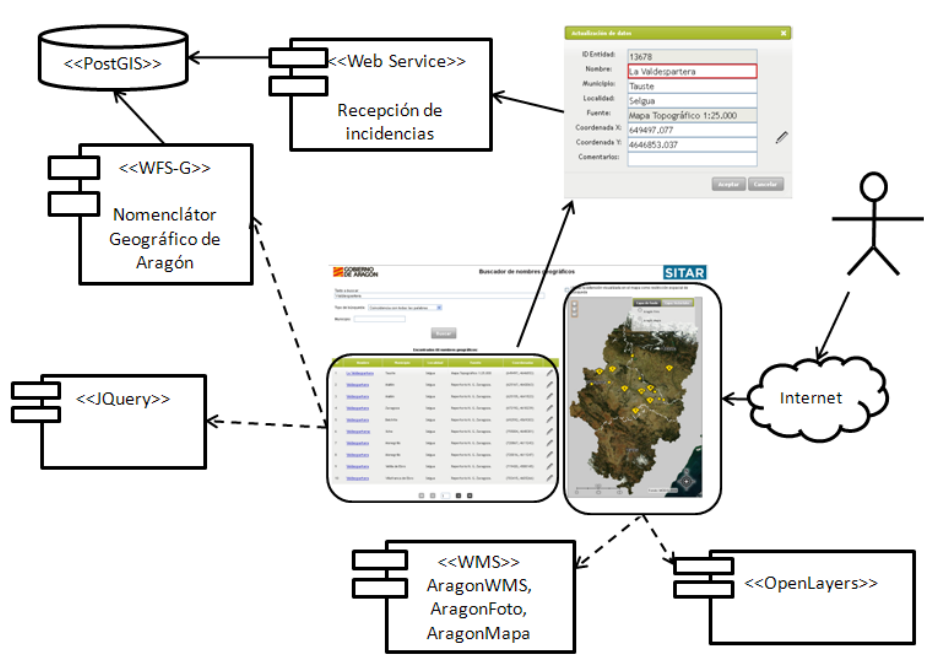

Figura 4. Diagrama de la arquitectura de despliegue del buscador de topónimos

La aplicación cliente ofrece la posibilidad de realizar búsquedas de tres tipos: coincidencia exacta, coincidencia con alguna de las palabras o coincidencia con todas las palabras. Los resultados se muestran en formato tabular paginado cada 10 resultados, con un número máximo de 200 resultados. Para aquellos topónimos georreferenciados localizados, se incluye una capa vectorial sobre el mapa que los representa, destacando los que se están visualizando en la página actual.

\section{Difusión e integración de la herramienta y de la base de datos toponímica}

Esta primera versión de la base de datos de nombres geográficos está puesta a disposición del ciudadano -y especialmente de los infomediarios - para que sea reutilizada en su beneficio en el ámbito que se desee conforme a unas condiciones de uso poco restrictivas. De este modo, puede servir de referencia para cualquier fuente oral o escrita que requiera la inclusión de nombres geográficos (Bernabé-Poveda, 2012).

Para ello, se ha incorporado para su descarga en la plataforma SITAR, dentro de la zona reservada para la descarga de información territorial, y se espera que esté también disponible en el portal de datos abiertos del Gobierno de Aragón, denominado Aragón Open Data y accesible a través de la dirección http://opendata.aragon.es.

En los próximos meses se irá actualizando esta base de datos y se irán generando sucesivas versiones que corrijan los errores detectados y que incorporen aquellas aportaciones realizadas a través del buscador. Por lo tanto, no es un producto fijo y cerrado, sino que se concibe como un producto en constante evolución cuyo producto final es el Nomenclátor Geográfico de Aragón.

Además, se va a realizar una integración de los procesos de búsqueda en diversas aplicaciones que requieran de esta funcionalidad. El primer caso de aplicación será el conjunto de aplicaciones web cartográficas que conforman la plataforma SITAR, tales como el visor 2D, la cartoteca o el visor de Régimen Jurídico, entre otros. Para que se conozca la existencia de esta herramienta y sus posibilidades de integración con otras posibles herramientas informáticas, está previsto realizar jornadas de difusión tanto internas como externas al Gobierno de Aragón a corto y medio plazo.

\section{Corresponsabilidad administración pública-ciudadano}

En los últimos tiempos, la tendencia es que casi todas las administraciones públicas han venido realizado un proceso de apertura de los datos que generan y que custodian; este proceso ha sido impulsado esencialmente tras la aprobación de la Ley 37/2007, de 16 de noviembre, sobre reutilización de la información del sector público. El caso descrito en el presente artículo acerca del Buscador de nombres geográficos es otro ejemplo de aplicación de dichas prácticas por parte del Gobierno de Aragón.

Sin embargo, como se ha descrito en el apartado tercero, la apertura, supervisión y mejora continua de los datos que obran en poder de la Administración no debería ser una tarea con responsabilidad exclusiva de la Administración pública, sino que también debería residir una pequeña parte de responsabilidad en el ciudadano. Sólo mediante la cooperación entre ambas esferas se logrará un producto final con un grado de calidad óptimo.

Por el lado de la administración pública, no basta con ofrecer alternativas básicas para obtener la cooperación ciudadana tales como el tradicional formulario en papel a presentar en un registro oficial; es necesario ofrecer cauces completos y sencillos de utilizar e integrados en los procedimientos de gestión de la información, si se desea aspirar a lograr una participación ciudadana efectiva y convertirse en un gobierno abierto y transparente. En definitiva, es necesario que el ciudadano perciba que se le valora y que se tiene en consideración el potencial que puede ofrecer para que se sienta involucrado en la tarea. Se debe no sólo solicitar su participación, sino que además se le debe reconocer sus aportaciones, para que se sienta partícipe de esta tarea de la que es corresponsable. 
Por el contrario, el ciudadano debe ser consciente de las limitaciones a las que está sometida la actividad de las distintas administraciones públicas y que éstas no disponen de recursos ilimitados para el ejercicio de sus funciones. Fomentar la conciencia cívica de cada uno de los ciudadanos en una sociedad moderna debe ser un reto a conseguir si se desea lograr una sociedad avanzada y acorde con las circunstancias actuales. Las Tecnologías de la Información y las Comunicaciones (TIC) -y, en este caso de las Tecnologías de la Información Geográfica (TIG), dado el componente geográfico de la información- ofrecen herramientas para lograr este reto y brindan mecanismos inimaginables hace años, pero no bastan por sí mismas para conseguirlo.

\section{Conclusiones y trabajo futuro}

En los apartados anteriores se ha descrito el caso de una aplicación web cartográfica habitual en una IDE a la cual se le ha aplicado una perspectiva más amplia: se la ha dotado de los elementos necesarios para convertirla en una herramienta colaborativa para la participación ciudadana. De este modo, se aspira a que todo ciudadano colabore con la administración pública competente por razón de la materia, en este caso el Gobierno de Aragón, para mejorar y ampliar la base de datos toponímica que almacena.

Los próximos meses servirán para valorar la correcta implantación y acogida de la herramienta, pero se espera que sea positiva. Las primeras pruebas que se han realizado con un subconjunto reducido de usuarios han permitido perfilar y corregir algunos aspectos, de carácter ergonómico, para que sea más sencilla de utilizar.

El resultado de la aplicación práctica del buscador de nombres geográficos implica la publicación en sí de un producto del que adolecía el Gobierno de Aragón; el Nomenclátor Geográfico de Aragón. De este modo, al margen de cumplir con uno de los objetivos planificados dentro del Plan Cartográfico de Aragón 2013-2016, se espera poder integrar el Nomenclátor con otros nomenclátores disponibles para realizar una búsqueda distribuida, ya sea mediante la técnica de harvesting o mediante la técnica de distribución de datos (Laborda, 2009).

El resultado satisface al equipo técnico encargado del desarrollo no sólo por el aspecto funcional, sino porque además cumple las expectativas de rendimiento y tiempos de respuesta, así como por las tareas de gestión y mantenimiento de los datos almacenados y ofrecidos por la aplicación. El proceso de atención y gestión de las propuestas de incidencias y aportaciones por parte de los usuarios también cumplen con los requisitos planteados y no suponen una sobrecarga excesiva de trabajo para su resolución por parte de los encargados de la plataforma.

La base de datos de nombres geográficos cuenta con un importante volumen de registros toponímicos y está a disposición del ciudadano para su posible reutilización posterior. Además, se ha previsto un protocolo de generación de versiones posteriores, que incorporen las correcciones detectadas a lo largo de los meses.

En definitiva, el resultado de los trabajos ha permitido dotar a la Comunidad Autónoma de Aragón de un valioso conjunto de datos susceptible de generar valor en el futuro; y se ha integrado al ciudadano en procesos hasta la fecha reservados en exclusiva a los encargados de la gestión de la plataforma SITAR, siendo el germen de futuras iniciativas similares en otros ámbitos de aplicación.

\section{Referencias}

Ayuda Bosque, M.I.; Pinilla Navarro, Vicente; Sáez Pérez, Luis Antonio (2000). El problema de la despoblación en Aragón: causas, características y perspectivas. // Boletín de la Asociación de Demografía Histórica. 18:1 (2000)137-175.

Benito Moliner, M. (2000). Pueblos del Alto Aragón. http://www.aragob.es/edycul/patrimo/etno/pueblos/indice .htm (2013-04-04).

Bernabé-Poveda, Miguel Á; López-Vázquez, Carlos M. (eds.) (2012). Fundamentos de las Infraestructuras de Datos Espaciales (IDE). Madrid: UPM Press, 2012.

Centro Nacional de Información Geográfica (2005). Toponimia: Normas para el MTN25. Conceptos básicos y terminología. Madrid: Ministerio de Fomento. Publicación técnica $\mathrm{n}^{\circ}$ 42. http://www.fomento.gob.es/NR/rdonlyres/ C7B3628F-1BFB-431E-A7FA-99A97C2A9ECC/28709/ NormasToponimiaparaMTN25.pdf (2013-04-04).

Comisión Especializada de Nombres Geográficos del Consejo Superior Geográfico. Centro Nacional de Información Geográfica (2011). Directrices Toponímicas de Uso Internacional para Editores de Mapas y otras Publicaciones. Madrid: Consejo Superior Geográfico. http://www.ign.es/ign/resources/acercaDe/marcoNormati vo/Directrices_toponimicas.pdf (2013-04-04).

Fernández Marcos, Vicenta (1981). Contribución al estudio de la toponimia en la provincia de León. // Tierras de León: Revista de la Diputación Provincial. 21:9 (1981) 97-116. http://www.saber.es/web/biblioteca/libros/tierrasde-leon/html/43/7contribucion.pdf (2013-03-26).

Hernández, Félix José (2008). Búsquedas inteligentes de toponimia. // V Jornadas Técnicas de la Infraestructura de Datos Espaciales de España: Tenerife, 5-7 noviembre. http://www.idee.es/resources/presentaciones/JIDE E08/ARTICULOS_JIDEE2008/articulo45.pdf (2013-0326).

Jones, Rosie; et al. (2006). Geographic Intention and Modification in Web Search. // International Journal of Geographical Information Science. 22:3 (2008) 1-20. 
http://www.cs.cmu.edu/ rosie/papers/geoQueryRewrites 2007.pdf (2013-03-31).

Kerfoot, Helen; et al. (2007). Manual para la normalización nacional de nombres geográficos. New York: Naciones Unidas. http://unstats.un.org/unsd/publication/seriesm/se riesm_88s.pdf (2013-03-31)

Laborda, C.; et al. (2009). Una aproximación a la búsqueda distribuida de topónimos. // VI Jornadas de la Infraestructura de Datos Espaciales de España: Murcia, 1-6 noviembre. http://iaaa.cps.unizar.es/curriculum/09-OtrasPublicaciones-Congresos/cong 2009 JIDEE Aproxima cion.pdf (2013-03-31).
Terrado Pablo, Javier (1999). Metodología de la investigación en toponimia. Zaragoza: Javier Terrado Pablo.

Ubieto Arteta, A. (1972). Toponimia Aragonesa Medieval. Zaragoza: Anubar.

Ubieto Arteta, A. (1984). Historia de Aragón: Los pueblos y los despoblados. Zaragoza: Anubar.

Enviado: 2013-05-21. Segunda versión: 2013-06-19. Aceptado: 2013-08-22. 